\title{
AUTOREGULATION OF BRAIN CIRCULATION IN PROGRESSING CARDIAC FAILURE AND ITS RELATION TO THE APPEARANCE OF SEIZURE READINESS
}

\author{
M.L. Mamalyga', L.M. Mamalyga ${ }^{2}$ \\ ${ }^{1}$ Bakulev Scientific Center of Cardiovascular Surgery, Moscow, Leninsky Prospekt 8, Russia \\ 2Department of Anatomy and Human and Animal Physiology of Institute Biology and Chemistry of \\ Moscow State University of Education, Moscow, Malaya Pirogovskaya St.1-1, Russia
}

\begin{abstract}
On the early stage of cardiac decompensation, the blood flow in common carotid and basilar arteries does not change, however the seizure readiness (SR) of animals increases. The preserved reaction on hypercapnic and compression tests allows us to stipulate that the increased SR is not related to the circulatory brain disorders. Progressive aggravation of cardiac failure (CF) leads to the severe stage of decompensation accompanied by decreased blood flow in common carotid and basilar arteries, as well as increases SR. At the same time the metabolic cascade of autoregulation is areactive and myogenic is significantly decreased. Ineffective operation of heart in different stages of heart failure shows not the same effect or backup possibilities for cerebral hemodynamic autoregulation affecting the formation and aggravation of SR. The increased SR in cardiac failure is not always caused by brain ischemia.
\end{abstract}

Keywords: autoregulation of brain circulation, cardiac failure, seizure readiness

\section{INTRODUCTION}

Multiple clinical studies means that in significant part of people suffering of chronic heart failure the cerebral hemodynamic disorders appear to make the disease outcome especially unfavorable $(1,2)$. Despite the brain having the mechanisms that ensure the autoregulation of cerebral blood flow, their compensation capacity eventually exhausts during aggravation of heart failure. The opinions of authors on associated progressive heart dysfunction and possibility for autoregulation of cerebral hemodynamic on different stages of cardiac decompensation differ. The results of a number of studies show the presence of positive correlation between cardiac index and cerebral hemodynamic $(2,3)$. However, the other works display no correlation between left ventricle ejection fraction and cerebral circulation (4). Thus the authors postulate the concept of cerebral circulation being independent of cardiac discharge.

Also heart failure is viewed not only as a risk factor for cerebral hemodynamic disorders, but also for related neurologic diseases that increase seizure readiness $(1,5,6)$. Thus for effective treatment of the patients with cardiocerebral seizure syndrome it is of interest to study the contingency of cerebral hemodynamic in progressing heart failure and the appearance of cerebral seizure readiness (SR). The most common opinion on this issue is that the reason for increased SR in heart failure is hypoxia caused by cerebral hypocirculation.

Study objective - to study the particularities in changes of autoregulation of cerebral hemodynamic depending on the degree of heart activity decompensation in progressing heart failure and to evaluate its relation with the seizure readiness manifestation.

\section{MATERIALS AND METHODS}

Experiments are performed on white male Wistar rats with the weight 200-220 g. All the studies were performed in strict conformity with the primary bioethics "Regulations on Studies with Test 
Animals", "International Recommendations on Medical and Biological Tests with Animals".

\section{Cardiac failure model}

The studies used doxorubicin-induced cardiac failure model which is the most adequate for reproducing of progressive heart failure of different degree of severity $(7,8)$. The animals underwent intraperitoneal infusions of doxorubicin by Farmohemi (Netherlands) in total dose $20 \mathrm{mg} / \mathrm{kg}$ separated into 5 weekly injections. Doxorubicin does not pass the hematoencephalic barrier. The control animals were treated with the respective doses of natural saline. US-investigations allowed us to study the effect of progressing heart failure on cerebral hemodynamic in three groups of animals: I - with compensated heart failure (CHF); II - with early stage of decompensation (ESD); III - with severe stage of decompensation (SSD).

\section{Ultrasound Doppler study of vessels}

For detection of compensated and decompensated heart failure stages each three days using echocardiography (echocardiograph Mindray M5, sensor $10 \mathrm{MHz}$, Mindray, China) the cardiac output (CO) was measured on blood flow velocity in pulmonary artery. The adequate cerebral perfusion in different stages of heart failure was studied based on the data of linear blood flow velocity during transcranial Doppler sonography of basilar artery.

The study was performed via scanning in color and pulse-wave Doppler mode. The quantitative evaluation of spectrum of Doppler shift was performed considering the parameters of peak systolic (Vps), maximum end diastolic (Ved), and time-averaged maximum velocity (TAMX). Also there were calculated peripheral resistance index (RI), pulsation index $(\mathrm{PI})$, systole-diastole ratio $(\mathrm{S} / \mathrm{D})$. Diameter of the left common carotid artery was measured based on the image in B-mode, color and energy Doppler. For the same artery the volumetric blood flow (VBF) was also determined.

\section{Functional probes}

For objective evaluation of potential capacity for autoregulation of cerebral hemodynamic the clinical practice widely uses hypercapnic (HT) and compression (CT) functional load tests, activating respectively metabolic and myogenic autoregulation mechanisms (9). The criteria for evaluation of potential capabilities of the mechanism is the "flexibility" range of cerebral hemodynamic system in response to short-term increase of $\mathrm{CO}_{2}$ content in blood or short-term compression of common carotid arteries. The main advantage of use of functional probes is that they allow to minimize the individual differences and evaluate the direction of changes.

In the animals in control and test groups the effects of hypercapnic test were evaluated after dosed inhalation of $6 \%$ mixture of carbon dioxide with air for 1.5 minutes. For the control of $\mathrm{CO}_{2}$ and $\mathrm{O}_{2}$ in inhaled air we used the capnograph "CARDEX MAR-02" (CARDEX LLC, Russia) and oxygen monitor "OxiQuant S Plus". The compression test was performed via short-term (5 s) compression of left common carotid artery (CCA).

The SR level in each animal was evaluated using common penthylentetrazole (PTZ) test (10). The criteria of SR evaluation was the individual threshold dose of PTZ (in $\mathrm{mg} / \mathrm{kg}$ body mass) capable of causing clonic-tonic seizures. For this purpose, using infusomat (Braun Perfusor Compact, Germany) the rats were given intravenous infusion of $1 \%$ PTZ solution (Sigma, USA) at the rate of 0.1 $\mathrm{ml} / \mathrm{min}$ till manifestation of external signs of increased seizure readiness (expressed anxiety increase, frequent grooming, clonus of face and then of back etc.). Then the seizure reactions developed spontaneously. During the test the evaluation was performed for total seizure duration and the duration of clonic and tonic phases.

\section{Magnetic resonance imaging (MRI)}

The studies were performed using the tomograph Bruker Biospec 70/30 with magnetic field inductivity $7 \mathrm{Tl}$ and induction coil diameter $72 \mathrm{~mm}$. The MRI-scanning protocol included T1- and T2weighted images in coronary and axial views (PS - pulse sequence SE - spine echo and RARE- relaxation contrast enhancement, slice thickness 1.5 $\mathrm{mm}$, resolution $0.1 \mathrm{~mm} /$ pix), angiography before and after the use of contrast (Magnevist Gd-DTPA). The images were processed using the software for MRI-images: ImageJ, MRIcron, 3D-DOCTOR. Also the studies were performed for functional capacity of the heart on the different heart failure stages: EDV - end diastolic volume, ESV - end systolic volume, SV- stroke volume, EF - left ventricle ejection fraction.

Statistical analysis was performed using the software Statistica 6.0. Study values were determined as direct average with standard error average $(\mathrm{M} \pm \mathrm{m})$. Validity of differences between the average values of study parameters in animals in control and test animals was evaluated using Student 
t-test. Statistically significant result was considered the value $\mathrm{P}<0.05$. The number of animals in each group is given in tables.

\section{RESULTS}

Ultrasound studies of blood flow in pulmonary artery, as well as functional MRI shoes that within $12 \pm 1.07$ days after the end of doxorubicin infusion the hemodynamic parameters remain on control level (Table 1). However the adequate minute blood flow volume was supported via increase $(\mathrm{P}<0.05)$ of heart rate (HR) signifying the compensated heart failure.

In $20 \pm 1.51$ days $92 \%$ of animals displayed the decompensate heart failure with decreased SV, left ventricle $\mathrm{EF}$ and $\mathrm{CO}$, while ESV and EDV increase by $139 \%$ and $45 \%$ respectively. However the average VBF in carotid artery did not change. The stage was marked by us as early heart failure.

In $35 \pm 2.59$ days after the end of doxorubicin infusion $95 \%$ of animals show severe hemodynamic failure. Clinical confirmation of severe heart failure is the appearance of liquid in pericardium, pleural cavity and abdominal cavity. Two animals died on this stage.

Transcranial Doppler scanning did not show the changes in cardiopulmonary circulation (Vps and Ved) in basilar artery of animals with CHF and ESD (Table 2) signifying the absence of brain circulation disorders. Also, the adequate response of basilar artery of animals with CHF on hypercapnic

TABLE 1. Hemodynamic changes at different stages of progressive heart failure

\begin{tabular}{|c|c|c|c|c|c|c|c|}
\hline Stage of $\mathrm{HF}$ & $\mathrm{CO}, \mathrm{ml} / \mathrm{min}$ & $\mathrm{ESV}, \mathrm{ml}$ & $\mathrm{EDV}, \mathrm{ml}$ & $\mathrm{SV}, \mathrm{ml}$ & $\mathrm{EF}, \%$ & $\begin{array}{c}\mathrm{HR}, \\
\text { beats } / \mathrm{min}\end{array}$ & $\begin{array}{c}\text { VBF of the CCA } \\
(\mathrm{ml} / \mathrm{min})\end{array}$ \\
\hline Control $(\mathrm{n}=15)$ & $199 \pm 13.9$ & $0.33 \pm 0.03$ & $0.85 \pm 0.07$ & $0.53 \pm 0.04$ & $62 \pm 4.59$ & $274 \pm 18.9$ & $12.7 \pm 1.07$ \\
\hline CHF $(\mathrm{n}=14)$ & $190 \pm 13.7$ & $0.36 \pm 0.03$ & $0.82 \pm 0.06$ & $0.44 \pm 0.03$ & $55 \pm 4.45$ & $\begin{array}{c}363 \pm 28.0 \\
\mathrm{P}<0.05\end{array}$ & $11.3 \pm 0.93$ \\
\hline ESD $(\mathrm{n}=14)$ & $\begin{array}{c}146 \pm 12.0 \\
\mathrm{P}<0.05\end{array}$ & $\begin{array}{c}0.79 \pm 0.06 \\
\mathrm{P}<0.001\end{array}$ & $\begin{array}{c}1.23 \pm 0.09 \\
\mathrm{P}<0.01\end{array}$ & $\begin{array}{c}0.37 \pm 0.03 \\
\mathrm{P}<0.01\end{array}$ & $\begin{array}{c}33 \pm 2.74 \\
\mathrm{P}<0.001\end{array}$ & $\begin{array}{c}385 \pm 31.2 \\
\mathrm{P}<0.01\end{array}$ & $10.6 \pm 1.01$ \\
\hline SSD $(\mathrm{n}=12)$ & $\begin{array}{c}111 \pm 8.43 \\
\mathrm{P}<0.001\end{array}$ & $\begin{array}{c}1.02 \pm 0.09 \\
\mathrm{P}<0.001\end{array}$ & $\begin{array}{c}1.26 \pm 0.10 \\
\mathrm{P}<0.01\end{array}$ & $\begin{array}{c}0.27 \pm 0.02 \\
\mathrm{P}<0.001\end{array}$ & $\begin{array}{c}21 \pm 1.62 \\
\mathrm{P}<0.001\end{array}$ & $\begin{array}{c}378 \pm 27.2 \\
\mathrm{P}<0.01\end{array}$ & $\begin{array}{c}8.2 \pm 0.68 \\
\mathrm{P}<0.01\end{array}$ \\
\hline
\end{tabular}

TABLE 2. Hemodynamic changes in the basilar artery at different stages of progressive heart failure

\begin{tabular}{|c|c|c|c|c|c|c|c|}
\hline $\begin{array}{c}\text { № } \\
\Pi / \Pi\end{array}$ & Воздействие & Vps, cm/c & Ved, $\mathrm{cm} / \mathrm{c}$ & TAMX, cM/c & $S / D$ & $\mathrm{PI}$ & RI \\
\hline 1 & Control $(n=15)$ & $46 \pm 3.17$ & $25 \pm 1.85$ & $33 \pm 2.11$ & $1.88 \pm 0.14$ & $0.65 \pm 0.04$ & $0.46 \pm 0.03$ \\
\hline 2 & CHF $\quad(n=14)$ & $42 \pm 3.15$ & $22 \pm 1.89$ & $30 \pm 2.55$ & $1.92 \pm 0.17$ & $0.67 \pm 0.06$ & $0.48 \pm 0.04$ \\
\hline 3 & $\operatorname{ESD}(n=14)$ & $52 \pm 3.84$ & $28 \pm 2.16$ & $\begin{array}{l}41 \pm 2.75 \\
P<0.05\end{array}$ & $1.88 \pm 0.15$ & $0.59 \pm 0.05$ & $0.47 \pm 0.04$ \\
\hline 4 & $\operatorname{SSD}(n=12)$ & $\begin{array}{l}35 \pm 2.83 \\
P<0.05\end{array}$ & $\begin{array}{l}17 \pm 1.50 \\
P<0.01\end{array}$ & $\begin{array}{l}23 \pm 1.61 \\
P<0.01\end{array}$ & $2.11 \pm 0.19$ & $\begin{array}{c}0.81 \pm 0.06 \\
P<0.05\end{array}$ & $0.53 \pm 0.03$ \\
\hline 5 & HT control $(n=15)$ & $\begin{array}{l}110 \pm 9.24 \\
P<0.001\end{array}$ & $\begin{array}{c}65 \pm 5.91 \\
P<0.001\end{array}$ & $\begin{array}{c}83 \pm 6.81 \\
P<0.001\end{array}$ & $1.68 \pm 0.12$ & $0.54 \pm 0.04$ & $0.40 \pm 0.03$ \\
\hline 6 & $\mathrm{HT}$ in $\mathrm{CHF} \quad(n=14)$ & $\begin{array}{c}101 \pm 9.19 \\
P<0.01\end{array}$ & $\begin{array}{c}64 \pm 5.44 \\
P<0.001\end{array}$ & $\begin{array}{c}77 \pm 6.47 \\
P<0.001\end{array}$ & $1.58 \pm 0.14$ & $\begin{array}{c}0.48 \pm 0.03 P \\
<0.01\end{array}$ & $\begin{array}{c}0.37 \pm 0.03 \\
P<0.05\end{array}$ \\
\hline 7 & $\mathrm{HT}$ in ESD $(n=14)$ & $\begin{array}{l}60 \pm 5.04 \\
P<0.05\end{array}$ & $\begin{array}{l}33 \pm 2.37 \\
P<0.05\end{array}$ & $38 \pm 2.31$ & $1.85 \pm 0.17$ & $0.72 \pm 0.06$ & $0,46 \pm 0.04$ \\
\hline 8 & $H T$ in SSD $(n=12)$ & $50 \pm 3.85$ & $27 \pm 2.19$ & $34 \pm 2.65$ & $1.83 \pm 0.18$ & $0.66 \pm 0.05$ & $0.45 \pm 0.04$ \\
\hline 9 & CT control $(n=15)$ & $\begin{array}{c}169 \pm 14.12 \\
P<0.001\end{array}$ & $\begin{array}{c}97 \pm 9.41 \\
P<0.001\end{array}$ & $\begin{array}{c}127 \pm 11.18 \\
P<0.001\end{array}$ & $1.73 \pm 0.13$ & $0.56 \pm 0.05$ & $0.42 \pm 0.03$ \\
\hline 10 & $\mathrm{CT}$ in $\mathrm{CHF}(\mathrm{n}=14)$ & $\begin{array}{c}150 \pm 13.22 \\
P<0.001\end{array}$ & $\begin{array}{c}89 \pm 7.74 \\
P<0.001\end{array}$ & $\begin{array}{c}112 \pm 10.31 \\
P<0.001\end{array}$ & $1.68 \pm 0.15$ & $0.54 \pm 0.04$ & $0.40 \pm 0.04$ \\
\hline 11 & CT in ESD $(n=14)$ & $\begin{array}{c}146 \pm 11.17 \\
P<0,001\end{array}$ & $\begin{array}{c}95 \pm 8.64 \\
P<0.001\end{array}$ & $\begin{array}{l}112 \pm 9.44 \\
P<0.001\end{array}$ & $1.53 \pm 0.13$ & $\begin{array}{c}0.45 \pm 0.04 \\
P<0.01\end{array}$ & $\begin{array}{c}0.35 \pm 0.03 \\
P<0.05\end{array}$ \\
\hline 12 & CT in SSD $(n=12)$ & $\begin{array}{c}77 \pm 6.54 \\
P<0.001\end{array}$ & $\begin{array}{c}48 \pm 3.71 \\
P<0.001\end{array}$ & $\begin{array}{c}65 \pm 5.52 \\
P<0.001\end{array}$ & $1.61 \pm 0.11$ & $\begin{array}{c}0.45 \pm 0.04 \\
P<0.01\end{array}$ & $0.38 \pm 0.02$ \\
\hline
\end{tabular}

$\mathrm{P}$ reflects the level of statistical significance of the changes between the studied parameters in animals with $\mathrm{HF}$ and a control group of rats. ${ }^{*}-\mathrm{P}<0.05 ; * *-\mathrm{P}<0.01 ; * * *-\mathrm{P}<0.001$ 

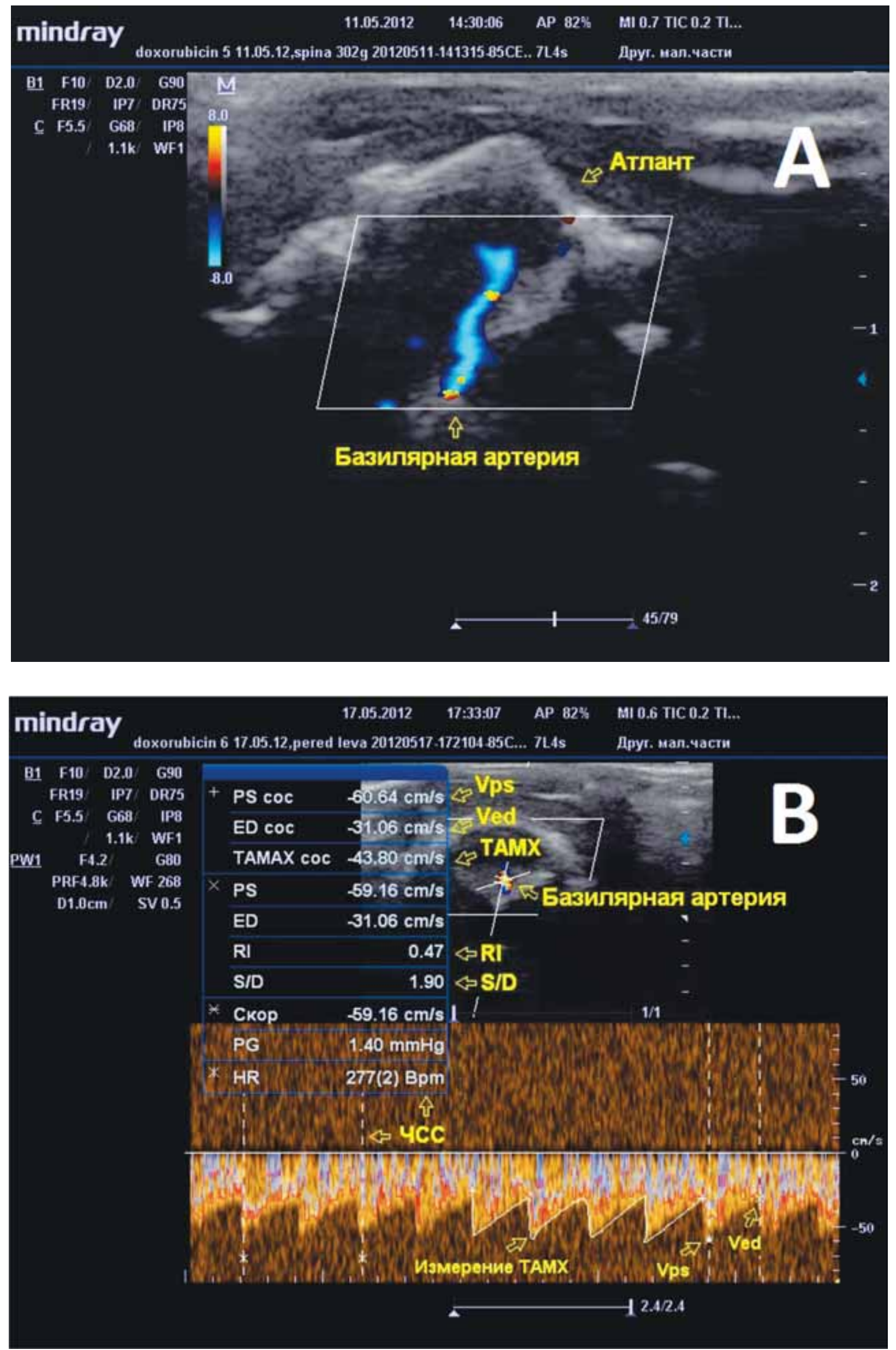

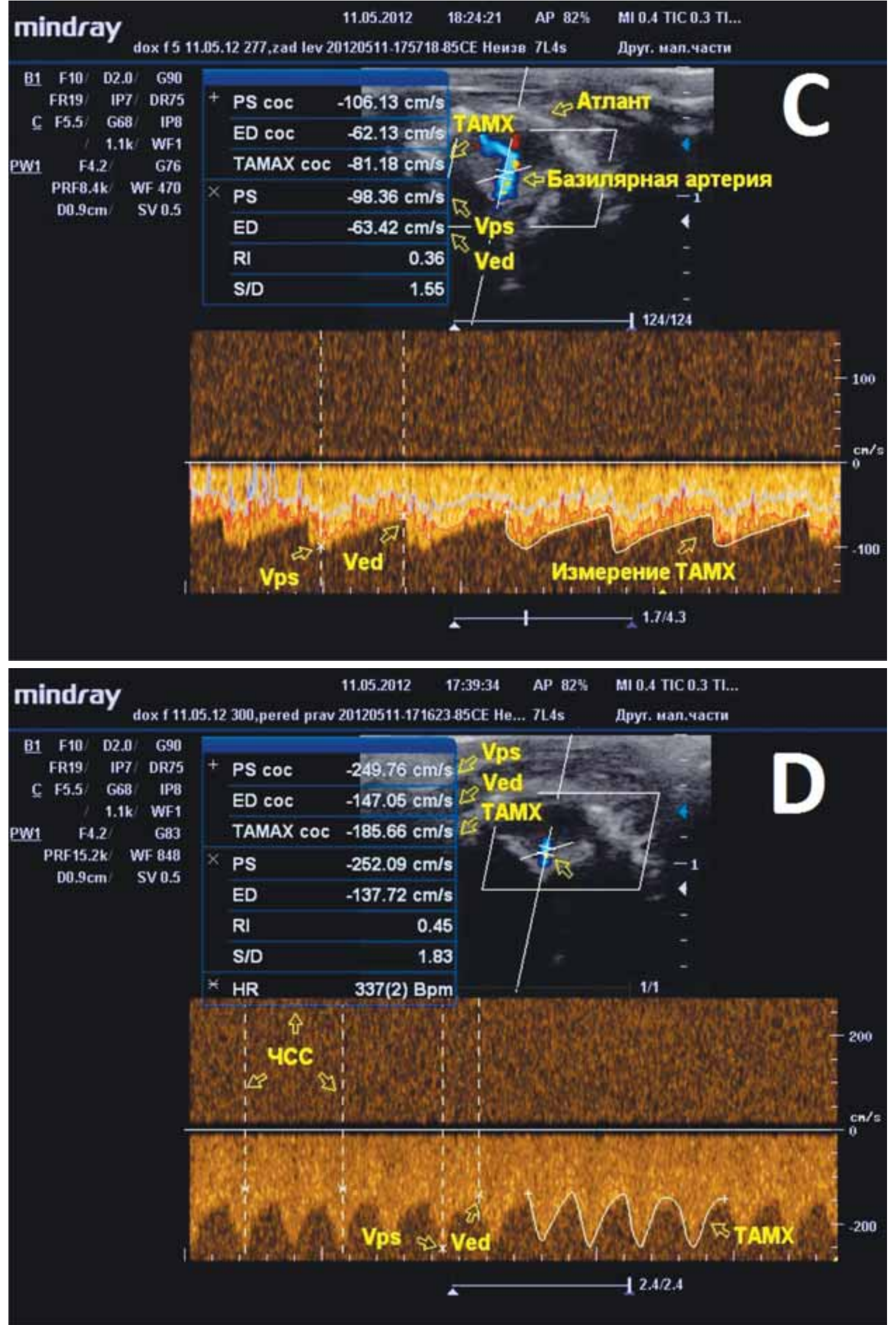

FIGURE 1. Transcranial doppler visualization of the basilar artery of the brain (A) and register blood flow at rest $(B)$, hypercapnic $(C)$ and compression $(D)$ functional tests in animals with compensated heart failure. 
and compression functional loads (Fig. 1) shows high reserves of metabolic and myogenic autoregulation cascades.

Lack of decreased blood flow velocity in common carotid and basilar arteries in ESD, as well as preserved reactivity to hypercapnic and compression tests allow to exclude the decreased oxygen support of the brain of those animals. At the same time, certain decrease (compared to CHF) of reserve of metabolic cascade of autoregulation, most likely, signifies overstrain of regulation systems in response to hypercapnic test. Also the increased blood flow in response to the compression test in ESD, accompanied by decrease in PI, is likely caused by the disorder in the vascular wall resistance that may serve as predictor for further decompensation of cerebral blood flow. This is supported by expressed decrease of cerebral blood flow detected in SSD.

Further aggravation of heart failure leads not only to progressing decrease of ejection fraction and decreased VBF in carotid artery, but also to the disorder in cerebral hemodynamic. In the circulation of basilar artery of the animals with SSD there is a decrease of Vps, Ved and TAMX, as well as increase of PI by $25 \%$ (Table 2), which reflects the decreased cardiopulmonary circulation and increased circulatory resistance. Also, the functional load tests detected not only the areactivity of metabolic cascade of autoregulation, but also the significant decrease in myogenic reserves of autoregulation.

Multiple clinical and experimental studies established that the decrease of cerebral hemodynamic below critical level is accompanied by increased seizure readiness (11). Due to this we were going to find out on which stage of heart failure the SR begins to increase and whether it is always related to hypoxia. Despite the absence of hemodynamic disorders in basilar artery of animals with CHF and ESD, their SR differed. The animals with CHF had the PTZ threshold dose not different from control, however the clonic phase during infusion of tis convulsant dose was $24 \%$ more long (Table 3 ).

The latter may signify that during the seizure attack appearing during CHF there is a decrease in capabilities of anti-seizure mechanisms which are activated during seizures and are directed towards weakening or stopping of the pathologic process. In the animals with ESD the seizure readiness increases by $26 \%$. At the same time the duration of clonic phase of seizure increases by $62 \%$, and tonic by $39 \%$. Thus despite the absence of circulatory brain disorders in ESD there is an increase in SR and increased duration of seizure attack, caused by threshold convulsant dose. Even higher increase in $\mathrm{SR}$ is detected in SSD.

\section{DISCUSSION}

Analysis of the literature data shows that presently there is no single opinion on interdependence of cerebral blood flow autoregulation and functional capabilities of heart in the different stages of heart failure. Discrepancy between the results of different authors (2-4), is likely caused by the fact that the examined patients' population had HF of different severity degree.

Results of our study showed that on the ESD stage the decrease in EF and SV does not cause the changes in cerebral circulation in basilar artery. However, further decrease in functional capability of the heart aggravates the heart failure and leads to SSD accompanied by the damage to the mechanisms of autoregulation and decreased hemodynamic in basilar artery. In the end it leads to hypoxic process that even further aggravate the autoregulation disorder, so even moderate hypoxia $\left(15 \% \mathrm{O}_{2}\right)$, active for 5 hours, gradually worsens cerebral autoregulation and decreases the cerebral circulation $(12,13)$. After adaptation to hypoxia the

TABLE 3. Threshold doses of pentylenetetrazol causing clonic-tonic seizures at different stages of progressive heart failure

\begin{tabular}{|c|c|c|c|c|}
\hline \multirow{2}{*}{ Stage of HF } & \multirow{2}{*}{ Doses of PTT $(\mathrm{mg} / \mathrm{kg})$} & \multicolumn{3}{|c|}{ Duration of the seizures (seconds) } \\
\cline { 3 - 5 } & & Total & Clonic phase & Tonic phase \\
\hline Control $(n=12)$ & $30.07 \pm 2.58$ & $9.68 \pm 0.72$ & $5.37 \pm 0.43$ & $4.31 \pm 0.40$ \\
\hline \multirow{2}{*}{ CHF $(n=10)$} & $27.97 \pm 2.17$ & $12.02 \pm 0.77$ & $6.98 \pm 0.59$ & $5.04 \pm 0.42$ \\
& $22.33 \pm 1.83$ & $14.69 \pm 1.33$ & $8.72 \pm 0.83$ & $5.97 \pm 0.55$ \\
\hline \multirow{2}{*}{ ESD $(n=11)$} & $P<0.05$ & $P<0.01$ & $P<0.01$ & $P<0.05$ \\
\hline SSD $(n=10)$ & $16.12 \pm 1.42$ & $21.17 \pm 2.17$ & $12.98 \pm 1.27$ & $8.19 \pm 0.91$ \\
& $P<0.01$ & $P<0.001$ & $P<0.001$ & $P<0.01$ \\
\hline
\end{tabular}


cerebral autoregulation of cerebral blood flow also remains disrupted $(14,15)$.

Thus we may consider that decreased cardiopulmonary circulation in basilar artery in SSD, caused by damaged autoregulation mechanisms, causes in the brain the aggravating hypoxic state accompanied by progressive increase in seizure readiness. Due to this we have a question on the reasons for increase of seizure readiness during adequate cerebral hemodynamic and rather high reserve possibilities for autoregulation in animals with ESD.

The reason for this may be the vegetative imbalance lying on the basis of pathogenesis of heart failure. This is confirmed by the data on influence of vegetative activity on SR. The efferent stimulation of nervus vagus during seizures in rats decreases the seizure activity, frequency of heart rate, blood pressure and cerebral circulation (16). Mentioned changes in cardiovascular system caused by the seizure attacks are mediated via vegetative hypothalamus region (17). Also during heart failure the hypothalamus has the increase in activity of neurosecretory neurons $(18,19)$. In opinion of authors this causes the increase in sympathic activity displayed both in heart dysfunction and in increased seizure readiness.

Study results allow us to consider that the increase in seizure readiness against the adequate cerebral blood flow in ESD is caused not by hypoxia but by neurovegetative imbalance which manifests earlier than the cerebral hemodynamic disorder.
Progressive increase in seizure readiness in SSD is related to the fact that vegetative disorders appearing in the stage of ESF are aggravated by hypoxicischemic processes. In its turn hypoxia causes the collection of agitating mediator glutamate in the brain (20), which is greatly caused by oxidative stress, production of active oxygen forms that decrease the content of glutamate transporters and decrease its activity $(21,22)$. Also hypoxia may cause the decrease in activity of inhibitory GABA-ergic mechanisms which is signified by decreased binding of $\left({ }^{3} \mathrm{H}\right)$ GABA to specific GABA-receptors in the brain cortex (12).

\section{CONCLUSIONS}

Ineffective operation of heart in early and severe heart decompensation stages differently affects the reserve capacity of cerebral hemodynamic autoregulation and the levels of brain seizure readiness. The increase of seizure activity appears as early as ESD stage, i.e. against the adequate cerebral blood flow. As the heart failure progresses, the significant decrease in cerebral hemodynamic is observed causing aggravating hypoxia and even more expressed decrease in seizure readiness than in ESD. The study results allow us to suggest that increase of brain seizure readiness in heart failure is not always caused by hypoxia, but its expression depends on the stage of cardiac decompensation.

\section{REFERENCES}

1. Ackerman R.H. Cerebral blood flow and neurological change in chronic heart failure. Stroke 2001; 32: 2462-2464.

2. Lepic T., Loncar G., Bozic B. et al. Cerebral blood flow in the chronic heart failure patients. Perspectives in Medicine 2012; 1:304-308.

3. Saha M., Muppala M.R., Castaldo J.E. et al. The impact of cardiac index on cerebral hemodynamics. Stroke 1993; 24:1686-1690.

4. Eicke B.M., von Schlichting J., Mohr-Ahaly S. et al. Lack of association between carotid artery volume blood flow and cardiac output. J. Ultrasound Med. 2001; 20: 1293-1298.

5. Halaris A. Comorbidity between depression and cardiovascular disease. Int. Angiol. 2009; 28: 92-99.

6. Montepietra S., Cattaneo L., Granella F. et al. Myocardial infarction convulsive and nonconvulsive seizures. Seizure 2009; 18: 379-381.

7. Gomes A.C., Falcao-Pires I., Pires A.L., Bras-Silva C., Leite-Moreira A.F. Rodent models of heart failure: an updated review. Heart Fail. Rev. 2013; 18:219-249.

8. Hensley M.L., Hagerty K.L., Kewalramani T. et al. American Society of Clinical Oncology 2008 clinical practice guideline update: use of chemotherapy and radiation therapy protectants. J. Clin. Oncol. 2009; 27:127-145

9. Zwiebel W.J. Introduction to Vascular Ultrasonography. 5rd ed. Philadelphia, Pa: WB Saunders Co; 2005.

10. Mamalyga M.L. Monoamine metabolism in the brain of rats with chronic heart failure of non ischemic origin. Neirokhimiya 2012; 1: $45-51$.

11. Engel J. Seizures and Epilepsy. Oxford University Press, 2013.

12. Viapiano M.S., Novara A.M.M., Plazas S.F., Bozzini C.E. Prolonged exposure to hypobaric hypoxia transiently reduces $\mathrm{GABA}_{\mathrm{A}}$ receptor number in mice cerebral cortex. Brain Res. 2001; 894: 31-36.

13. Nishimura N., Iwasaki K., Ogawa Y., Aoki K. Decreased steadystate cerebral blood flow velocity and altered dynamic cerebral autoregulation during 5-h sustained $15 \%$ O2 hypoxia. J. Appl. Physiol. 2010; 108: 1154-1161.

14. Iwasaki K., Zhang R., Zuckerman J.H. et al. Impaired dynamic cerebral autoregulation at extreme high altitude even after acclimatization. J. Cereb. Blood Flow Metab. 2011; 31: 283-292.

15. Lucas S.J.E., Burgess K.R., Thomas K.N. et al. Alterations in cerebral blood flow and cerebrovascular reactivity during 14 days at 5050 m. J. Physiol. 2011; 589: 741-753.

16. Hotta H., Watanabe N., Orman R., Stewart M. Efferent and afferent vagal actions on cortical blood flow and kainic acid-induced seizure activity in urethane anesthetized rats. Auton. Neurosci. 2010; 156: 144-148.

17. Stewart M. Autonomic consequences of seizures, including sudden unexpected death in epilepsy. In: Schwartzkroin Ph.A (eds.) 
Encyclopedia of Basic Epilepsy Research. ed. University of California, Davis, USA, 2009: 1289-1294.

18. Patel K.P., Zhang K., Kenney M.J. et al. Neuronal expression of Fos protein in the hypothalamus of rats with heart failure. Brain Res. 2000; 865: 27-34.

19. Zhang K., Zucker I. H., Patel K.P. Altered number of diaphorase (NOS) positive neurons in the hypothalamus of rats with heart failure. Brain Res. 1998; 786: 219-225

20. Parfenova H., Fedinec A., Leffler C.W. Ionotropic glutamate receptors in cerebral microvascular endothelium are functionally linked to heme oxygenase. J. Cereb. Blood Flow Metab. 2003; 23:190-197.

21. Boycott H.E., Dallas M., Boyle J.P. et al. Hypoxia suppresses astrocyte glutamate transport independently of amyloid formation. Biochem. Biophys. Res. Comm. 2007; 364: 100-104.

22. Dallas M., Boycott H.E., Atkinson L. et al. Hypoxia Suppresses Glutamate Transport in Astrocytes. J. Neuros. 2007; 27: 3946 -3955. 